\title{
Positive association between the plasma levels of 5-hydroxyindoleacetic acid and the severity of depression in patients with chronic obstructive pulmonary disease
}

\author{
Tomomi Sekiduka-Kumano', Tomotaka Kawayama1*, Kosuke Ito', Yoshihisa Shoji², Kazuko Matsunaga', \\ Masaki Okamoto ${ }^{1}$, Nobutaka Edakuni ${ }^{1}$, Haruki Imaoka', Naohisa Uchimura ${ }^{2}$ and Tomoaki Hoshino'
}

\begin{abstract}
Background: The role of plasma monoamines in patients with chronic obstructive pulmonary disease (COPD) with depression is unclear. To investigate monoamines in 20 depressed patients with COPD, the plasma concentrations of serotonin, 5-hydroxyindoleacetic acid (5-HIAA), homovanillic acid, and 3-methoxy-4-hydroxyphenylglycol (MHPG) were measured and compared with those in 50 non-depressed COPD patients, and also with 23 age- and gender-matched non-smokers and 13 smokers as non-depressed healthy controls.

Methods: Diagnosis of depression was assessed using the Centre for Epidemiologic Studies Depression Scale. Plasma concentrations of monoamines were measured by high-performance liquid chromatography.

Results: None of the depressed COPD patients had suicidal ideation. The plasma 5-HIAA level [median, (25\% and $75 \%$ quartiles)] in depressed COPD patients [6.8 $\mathrm{ng} / \mathrm{mL}$, (4.9 and 13.1)] was significantly higher than in non-depressed COPD patients [5.4, (4.2 and 7.5)] ( $p=0.022)$ and non-smokers [5.1 (3.8 and 7.2)] ( $p=0.041)$, but not smokers [4.7, (4.0 and 6.7)] ( $p>0.05)$. The plasma 5-HIAA level $(r=0.24, p=0.049)$ was significantly associated with the severity of depression in patients with COPD. The plasma MHPG level was significantly higher in depressed COPD patients ( $p=0.043$ ) than in smokers, but was not higher than that in non-depressed COPD patients or non-smokers, although the level of MHPG was not associated with the severity of depression.
\end{abstract}

Conclusion: The plasma 5-HIAA level is increased in depressed COPD patients. Plasma monoamines may be a good biomarker for detection of depression in patients with COPD.

Keywords: COPD, Monoamine, Depression

\section{Background}

Chronic obstructive pulmonary disease (COPD) is characterized by a chronic airflow limitation, and is recognized as a major health problem responsible for chronic morbidity and mortality worldwide [1]. Symptomatic COPD patients who have suffered previous repeated exacerbations have poor disease control and prognosis [2]. Improvement of symptoms

\footnotetext{
* Correspondence: kawayama_tomotaka@med.kurume-u.ac.jp 'Division of Respirology, Neurology, and Rheumatology, Department of Medicine 1, Kurume University School of Medicine, 67 Asahi-machi, Kurume 830-0011, Japan

Full list of author information is available at the end of the article
}

and prevention of exacerbations may contribute to an improvement of health-related quality of life (HRQOL) and lower mortality for patients with COPD.

COPD patients often have psychological disorders, including depression, and such patients tend to have more frequent exacerbations and a poor prognosis [3-7]. Recently, we demonstrated that depressed COPD patients had a lower HRQOL and more frequent exacerbations and hospitalizations those nondepressed COPD patients [3]. The severity of depression in COPD is closely associated with suicidal ideation $[8,9]$.

\section{Biomed Central}

(c) 2013 Sekiduka-Kumano et al.; licensee BioMed Central Ltd. This is an Open Access article distributed under the terms of the Creative Commons Attribution License (http://creativecommons.org/licenses/by/2.0), which permits unrestricted use, distribution, and reproduction in any medium, provided the original work is properly cited. 
It is well known that depressive symptoms are associated with dysfunction of brain monoaminergic neurons, and that the levels of serotonin (5-hydroxytryptamine [5-HT]) released in the brain are linked to a decrease in responsiveness to anti-depressants [10,11]. It is also well known that the functions of monoamine and monoamine oxidase are associated with smoking-related diseases $[12,13]$. However, the relationship between levels of plasma monoamines and their metabolites in patients with COPD-associated depression is still unclear.

In the present study, we analyzed serotonin metabolites to investigate possible biomarkers of depressed COPD patients. Plasma homovanillic acid (HVA), 3methoxy-4-hydroxyphenylglycol (MHPG), 5-HT, and 5hydroxyindoleacetic acid (5-HIAA) were measured in COPD patients who were depressed (depressed COPD) and COPD patients who were not depressed (non-depressed COPD), and also in age- and gender-matched non-depressed nonsmokers and smokers as controls.

\section{Methods}

\section{Participants}

The subjects of this study were outpatients or healthy volunteers. We randomly enrolled 70 patients with COPD, and recruited 36 age- and gender-matched healthy controls between September $1^{\text {st }} 2009$ and August $31^{\text {st }} 2011$ at the Chest Disease Center of Kurume University Hospital, Japan (Table 1). All of the patients analyzed had had stable COPD for at least 4 weeks prior to blood tests. None had received oral or injective corticosteroids or antibiotics for 4 weeks prior to the blood tests. Individuals with asthma, bronchiectasis, interstitial pneumonia, tuberculosis, pneumoconiosis, ischemic heart disease, chronic heart disease, renal or liver failure, active malignancies of any organs, sleep apnea syndrome, and a presence and history of psychological diseases such as major depression, bipolar disorder, or schizophrenia were excluded. Also excluded were patients who had been taking anti-depressants, and patients who had a history of lung volume reduction surgery, lung transplantation, or pneumonectomy. Patients with central nervous system disorders and cerebrovascular diseases were excluded on the basis of brain computed tomography (CT) or magnetic resonance imaging (MRI) examinations. Patients with COPD who were undertaking respiratory rehabilitation, or receiving long-term oxygen therapy and non-invasive positive pressure ventilation were excluded, because these treatments are thought to affect psychological status. We carefully excluded any subjects with renal function disorders (serum creatinine levels $>1.2 \mathrm{mg} / \mathrm{dL}$ ).

As reported previously $[14,15]$, the sample sizes for the patients with COPD and healthy controls were $>70$ and $>35$, respectively, in plasma levels of monoamines, when the sample ratio was $1: 2$ (power $=80 \%$; alpha error $=5 \%$; and beta error $=80 \%)$.

\section{Study protocol}

After the patients had provided written informed consent, information on age, gender, smoking status (current-, exor non-smoker), cumulative smoking history (pack-yrs), body mass index (BMI; weight/height $\left.{ }^{2}\right)$, comorbidities, and history of pharmacological treatments was obtained. Each subject underwent blood tests, spirometry, electrocardiography, chest radiography, chest high-resolution CT (HRCT), and brain CT or MRI. Spirometry and bronchodilator response tests were performed using an electronic spirometer (Chestgraph Jr HI-101, CHEST Ltd., Tokyo, Japan) in accordance with the American Thoracic Society (ATS) recommendations [16]. A metered-dose salbutamol (400 mcg/subject, GSK, Japan) inhaler was used as a bronchodilator, and bronchodilator response tests were performed before and $30 \mathrm{~min}$ after salbutamol inhalation. Predicted values of spirometry parameters were calculated according to the prediction equations of the Japanese Respiratory Society, as we have reported previously [17]. HRQOL was assessed using the validated Japanese St. George's Respiratory Questionnaire (SGRQ) $[18,19]$. The SGRQ contains three subscales (symptoms, activity, and impact), and the total score varies from 0 to 100 with a higher score indicating a worse health status [19]. Dyspnea was evaluated using the 5 -grade (0 to 4$)$ modified Medical Research Council (mMRC) dyspnea scale [20]. Arterial blood gas analysis was performed with each subject supine breathing room air. After assessing the SGRQ, the mMRC dyspnea scale, and the Centre for Epidemiologic Studies depression (CES-D) scale (Purchased from Saccess Bell Co., Ltd, Japan) for depression, all blood samples were taken between 9:00 and 10:00 AM following 10 minutes with the subjects supine. Samples were kept at $-80^{\circ} \mathrm{C}$ until analysis.

The study protocols (Approval No. 08091, May 29 ${ }^{\text {th }}$, 2009) were approved by the research ethics board of Kurume University and written informed consent was obtained from the internal review board and all participants.

\section{Diagnosis and severity of COPD}

Diagnosis and staging of COPD were in accordance with the Global Initiative for Chronic Obstructive Lung Disease (GOLD) guidelines [2], and included a postbronchodilator forced expiratory volume in 1 second/ forced vital capacity $\left(\mathrm{FEV}_{1} / \mathrm{FVC}\right)$ ratio of $<70 \%$, and $<200 \mathrm{~mL}$ and $<12 \%$ reversibility of $\mathrm{FEV}_{1}$ before and after bronchodilator administration. Patients with COPD who had a smoking history of 10 pack-yrs and over and also had emphysematous changes in the lungs were selected to carefully remove asthmatics. The emphysematous 
Table 1 Profiles of the four participant groups

\begin{tabular}{|c|c|c|c|c|}
\hline \multirow[t]{2}{*}{ Parameter } & \multicolumn{2}{|c|}{ Control subjects } & \multicolumn{2}{|c|}{ COPD patients } \\
\hline & Nonsmokers & Smokers & Non-depressed & Depressed \\
\hline Number of subjects & 23 & 13 & 50 & 20 \\
\hline Age (yr) & $66.7 \pm 9.0$ & $66.5 \pm 11.6$ & $68.5 \pm 7.2$ & $68.2 \pm 7.8$ \\
\hline Gender $^{\mathrm{a}}$ (no. of males; \%) & $15(65.2)$ & $12(92.3)$ & $47(94.0)$ & $14(70.0)$ \\
\hline Body mass index $\left(\mathrm{kg} / \mathrm{m}^{2}\right)$ & $22.1 \pm 2.4$ & $23.0 \pm 2.8$ & $21.9 \pm 3.1$ & $19.8 \pm 3.8^{\dagger}$ \\
\hline \multicolumn{5}{|l|}{ Smoking status ${ }^{\mathrm{a}}$} \\
\hline Non / Ex / Cu (no.) & $23 / 0 / 0$ & $0 / 4 / 9$ & $0 / 33 / 17$ & $0 / 14 / 6$ \\
\hline Smoke index (pack-yrs) & 0 & $40.0 \pm 18.2^{* * *}$ & $59.3 \pm 30.3^{* * *}$ & $52.2 \pm 27.0^{* * *}$ \\
\hline \multicolumn{5}{|l|}{ Comorbidities $^{a}$} \\
\hline Hypertension (no; \%) & $7(30.4)$ & $4(30.8)$ & $13(26.0)$ & $4(20.0)$ \\
\hline Diabetes (no; \%) & $5(21.7)$ & $1(7.7)$ & $11(22.0)$ & $6(30.0)$ \\
\hline Duration of COPD (yr) & N/A & N/A & $5.3 \pm 3.9$ & $5.5 \pm 4.4$ \\
\hline \multicolumn{5}{|l|}{ GOLD stage $e^{a}$} \\
\hline | / || / III / IV (no.) & N/A & N/A & $7 / 22 / 17 / 4$ & $2 / 4 / 8 / 6$ \\
\hline \multicolumn{5}{|l|}{ Lung function parameters } \\
\hline \multicolumn{5}{|l|}{ Before bronchodilator } \\
\hline FVC (L) & $3.5 \pm 0.9$ & $3.6 \pm 0.7$ & $3.4 \pm 0.8$ & $2.7 \pm 0.9^{* \neq \neq}$ \\
\hline$\% \mathrm{FVC}$ & $108.5 \pm 17.0$ & $105.6 \pm 19.3$ & $98.8 \pm 18.3$ & $86.4 \pm 22.2^{* *+}$ \\
\hline $\mathrm{FEV}_{1}(\mathrm{~L})$ & $2.6 \pm 0.6$ & $2.6 \pm 0.5$ & $1.5 \pm 0.6^{* * *+t+}$ & $1.1 \pm 0.7^{* * *+\dagger \dagger}$ \\
\hline$\% \mathrm{FEV}_{1}$ & $100.8 \pm 14.3$ & $94.9 \pm 20.1$ & $54.9 \pm 20.8^{* * *+t \dagger}$ & $44.8 \pm 24.9^{* * *+t+}$ \\
\hline $\mathrm{FEV}_{1} / \mathrm{FVC}(\%)$ & $77.2 \pm 6.9$ & $73.8 \pm 5.2$ & $44.4 \pm 12.7^{* * *+t \dagger}$ & $41.0 \pm 16.2^{* * *++\dagger}$ \\
\hline \multicolumn{5}{|l|}{ After bronchodilator } \\
\hline FVC (L) & $3.4 \pm 0.9$ & $3.5 \pm 0.7$ & $3.4 \pm 0.8$ & $2.6 \pm 1.0^{*+\neq}$ \\
\hline$\% \mathrm{FVC}$ & $108.0 \pm 17.4$ & $104.3 \pm 18.0$ & $99.3 \pm 18.5$ & $84.9 \pm 23.5^{* *+\neq}$ \\
\hline $\mathrm{FEV}_{1}(\mathrm{~L})$ & $2.7 \pm 0.6$ & $2.7 \pm 0.4$ & $1.6 \pm 0.6^{* * *++}$ & $1.2 \pm 0.7^{* * *++\dagger}$ \\
\hline$\% \mathrm{FEV}_{1}$ & $103.0 \pm 15.5$ & $96.5 \pm 19.9$ & $56.4 \pm 20.8^{* * *++\dagger}$ & $45.3 \pm 25.0^{* * *++\dagger}$ \\
\hline $\mathrm{FEV}_{1} / \mathrm{FVC}(\%)$ & $79.2 \pm 6.5$ & $76.0 \pm 5.9$ & $45.6 \pm 13.4^{* * *+t+}$ & $42.3 \pm 15.9^{* * *}+t \dagger$ \\
\hline Reversibility of FEV $1 \%$ ) & $2.2 \pm 4.5$ & $1.8 \pm 3.8$ & $3.3 \pm 5.3$ & $1.0 \pm 5.2$ \\
\hline \multicolumn{5}{|l|}{ Arterial blood gases } \\
\hline $\mathrm{PaO}_{2}$ (Torr) & $90.3 \pm 7.5$ & $92.9 \pm 5.9$ & $76.6 \pm 9.5^{* * *+t \dagger}$ & $71.9 \pm 13.9^{* * *++\dagger}$ \\
\hline $\mathrm{PaCO}_{2}$ (Torr) & $41.4 \pm 3.2$ & $41.7 \pm 3.5$ & $40.0 \pm 4.0$ & $44.6 \pm 7.1^{\neq}$ \\
\hline mMRC dyspnea scale & $0.0 \pm 0.0$ & $0.2 \pm 0.6$ & $1.0 \pm 1.1^{* * *+t+}$ & $2.1 \pm 1.6^{* * * t+t+\S}$ \\
\hline \multicolumn{5}{|l|}{ SGRQ } \\
\hline Total score (units) & $8.4 \pm 8.3$ & $15.7 \pm 12.0$ & $32.6 \pm 15.9^{* * *+\dagger}$ & $57.8 \pm 20.8^{* * *+++\bullet}$ \\
\hline Symptom score (units) & $19.6 \pm 13.4$ & $30.9 \pm 18.9$ & $40.8 \pm 21.3^{* *+1}$ & $66.2 \pm 16.7^{* * *+t+9}$ \\
\hline Activity score (units) & $6.0 \pm 7.1$ & $20.9 \pm 17.2$ & $42.9 \pm 24.1^{* * *++\dagger}$ & $68.2 \pm 29.7^{* * *++15}$ \\
\hline Impact score (units) & $6.2 \pm 10.0$ & $8.0 \pm 9.9$ & $21.3 \pm 13.9^{* *+\dagger}$ & $52.0 \pm 23.2^{* * *+t+19}$ \\
\hline CES-D scale & $1.7 \pm 2.9$ & $2.5 \pm 3.1$ & $8.5 \pm 5.2^{* * *+t \dagger}$ & $24.5 \pm 6.0^{* * *+t+9}$ \\
\hline \multicolumn{5}{|l|}{ Treatments for COPD } \\
\hline LAMA (no; \%) & 0 & 0 & $33(66.0)$ & $16(80.0)$ \\
\hline
\end{tabular}


Table 1 Profiles of the four participant groups (Continued)

LABA (no; \%)
ICS (no; \%)
SRT (no; \%)

changes were visually recognized as low-attenuation areas by chest HRCT [21].

\section{Diagnosis of depression}

Diagnosis of depression was assessed using the validated Japanese CES-D scale. The cut-off point for depression was CES-D >16 [22,23] and a CES-D score was assessed for each subject. The results of the CES-D score were not opened until this study was completed. Therefore, physicians did not know the psychological conditions of each subject. In this study, patients with a CES-D score of $>16$ were a subgroup of "depressed" COPD patients. For the purpose of this study, "depression" means possible "depression" as determined by the CES-D score.

\section{Measurement of plasma monoamine levels}

Plasma levels of 5-HT, 5-HIAA, HVA, and MHPG were measured by high-performance liquid chromatography (SRL Inc., Tokyo, Japan), as previously reported [15,24-26]. The lowest detection limits for 5-HT, 5-HIAA, HVA, and MHPG were $0.01 \mu \mathrm{g} / \mathrm{mL}, 1.8 \mathrm{ng} / \mathrm{mL}, 4.4 \mathrm{ng} / \mathrm{mL}$, and $3.2 \mathrm{ng} / \mathrm{mL}$, respectively, and for statistical analysis one half of the lowest value was assigned if the value was below the detection limit.

\section{Statistical analyses}

Data analyses were performed using JMP version 7 (SAS Institute, Inc., Cary, NC). Data for the subjects were expressed as the mean \pm standard deviation (SD), and data for plasma monoamine levels were expressed as the median, and $25 \%$ and $75 \%$ quartiles. Statistical analyses were performed using parametric Student's $t$ test for comparison between two groups, or one-way analysis of variance (ANOVA) and Tukey-Kramer test for multiple comparisons among four groups. Correlations were analyzed by parametric or non-parametric Spearman's tests. Differences between groups were evaluated using the chi-squared test for trend and Fisher's exact test. The level of significance was set at $\mathrm{P}<0.05$.

\section{Results}

\section{Subject characteristics}

A total of 106 subjects participated in the study and the characteristics of the four groups, namely the 23 nonsmokers and 13 smokers (as age- and gender-matched non-depressed healthy controls), and 50 non-depressed and 20 depressed COPD patients, are compared in Table 1. The depressed subjects without COPD were not enrolled in the study. None of participants, including the depressed COPD patients, had any history of attempted suicide.

The control subjects were 23 non-, 4 Ex-, and 9 current smokers, whereas the number of non-, Ex-, and current smokers were zero, 57, and 23 in patients with COPD $(\mathrm{p}<0.001)$. There was no significant difference in the smoke index among smokers, and non-depressed, and depressed COPD patients. There were no significant difference in the populations of subjects with hypertension and diabetes among nonsmokers, smokers, and, non-depressed, and depressed COPD patients ( $\mathrm{p}>0.05)$. All the subjects with hypertension had been taking antihypertensive medications whereas the numbers of nonsmokers, smokers, non-depressed, and depressed COPD patients taking anti-diabetes medications were $5,1,7$ and 4 , respectively.

In COPD, there was no significantly difference in duration of COPD between non-depressed and depressed COPD patients $(\mathrm{p}>0.05)$. The depressed COPD patients trended to have more progressive GOLD stages than the non-depressed patients but there was no significant difference in the populations of GOLD stage I (14\% vs $10 \%)$, II ( $44 \%$ vs $20 \%$ ), III ( $34 \%$ vs $40 \%$ ), and IV ( $8 \%$ vs $30 \%$ ) between two groups ( $\mathrm{p}>0.05$ by Chi-square test for trend).

Lung function tests showed that he depressed COPD patients had significantly lower FVC and \%FVC than the non-depressed patients both before and after bronchodilator use, although both depressed and non-depressed COPD patients had significantly lower $\mathrm{FEV}_{1}, \% \mathrm{FEV}_{1}$ and $\mathrm{FEV}_{1} / \mathrm{FVC}$ both before and after bronchodilator use than non-smokers and smokers, respectively (all $\mathrm{p}<0.001$ ). 
However, there was no difference in the reversibility of $\mathrm{FEV}_{1}$ after bronchodilator use among the four groups.

Arterial blood gas analysis showed that the depressed COPD patients $(\mathrm{p}<0.05)$ had significantly more severe hypercapnia than the non-depressed patients, whereas both depressed (both, $\mathrm{p}<0.001$ ) and non-depressed COPD patients (both, $p<0.001$ ) had significantly more severe hypoxia than the non-smokers and smokers, respectively.

Depressed COPD patients had significantly higher mMRC dyspnea scales $(\mathrm{p}<0.05)$ and lower HRQOL scores $(p<0.05)$ than the non-depressed patients, although both the depressed (both, $\mathrm{p}<0.001$ ) and non-depressed COPD patients (both, $\mathrm{p}<0.001$ ) had significantly higher MRC dyspnea scales and lower HRQOL scores than the nonsmokers and smokers, respectively.

In managements for COPD, all patients with COPD were receiving vaccinations for seasonal and $\mathrm{H} 1 \mathrm{~N} 1$ influenza virus and the numbers of depressed and non-depressed COPD patients who had been receiving pneumococcal vaccination within 5 yrs before recruitment were 13 and 8 , respectively. There was no significant difference in the ratio of regular use of ICS, LAMA, LABA, and SRT between non-depressed and depressed COPD patients $(\mathrm{p}=0.061$, $\mathrm{p}=0.387, \mathrm{p}=0.601$, and $\mathrm{p}=0.717$, respectively. The effects of ICS on psychological and mood status could not be directly determined, as the study was not designed to include a period for wash-out of each controller for COPD.

\section{Plasma monoamine levels}

Plasma 5-HIAA levels [median, (25\% and 75\% quartiles)] in the depressed COPD patients $[6.8 \mathrm{ng} / \mathrm{mL}$, (4.9 and 13.1)] were significantly higher than in the non-depressed patients [5.4, (4.2 and 7.5)] $(\mathrm{p}=0.022)$ and non-smokers [5.1 (3.8 and 7.2)] $(\mathrm{p}=0.041)$, respectively, but were not significantly higher than in the smokers [4.7, (4.0 and 6.7)] (Figure 1).

Median (25\% and 75\% quartiles) plasma 5-HT levels in the non-smokers, smokers, and non-depressed and depressed COPD patients were $0.06 \mu \mathrm{g} / \mathrm{mL}(0.04$ and 0.09 ), 0.05 (0.04 and 0.08), 0.06 (0.03 and 0.08), and 0.06 (0.02 and 0.09), respectively. The differences among the four groups were not significant (Figure 1).

Median (25\% and 75\% quartiles) plasma HVA levels in the non-smokers, smokers, and non-depressed and depressed COPD patients were $12.8 \mathrm{ng} / \mathrm{mL}$ (11.0 and 14.8), 11.8 (10.3 and 21.3), 14.8 (11.1 and 20.9), and 15.7 (9.9 and 22.2), respectively. The differences among the four groups were not significant (Figure 1).

Plasma MHPG level [median, (25\% and 75\% quartiles)] in the depressed COPD patients [6.8 $\mathrm{ng} / \mathrm{mL}$, (5.2 and 8.7)] $(\mathrm{p}=0.043)$ was significantly higher than in the smokers [4.6, (4.3 and 5.5)]. There was no significant difference in plasma MHPG level between the depressed COPD patients and either non-smokers [5.7 (4.7 and 7.2)] or non- depressed COPD patients [6.7, (4.1 and 8.2)] ( $\mathrm{p}>0.05)$, respectively (Figure 1).

To investigate seasonal effects in plasma 5-HIAA levels, plasma obtained in four seasons, spring (March-May), summer (June-August), fall (September-November), and winter (December-February), were measured. Number of all subjects and COPD patients in four seasons were 17 and 10,34 and 27, 25 and 16, and 30 and 17, respectively. There was no significant difference in median plasma 5HIAA [6.7 $\mathrm{ng} / \mathrm{mL}$ (5.1 and 7.6) in spring, $5.3 \mathrm{ng} / \mathrm{mL}(4.3$ and 7.4) in summer, $5.9 \mathrm{ng} / \mathrm{mL}$ (3.9 and 10.6) in fall, and $5.4 \mathrm{ng} / \mathrm{mL}$ (3.7 and 8.2) in winter, respectively, $\mathrm{p}>0.05$ ].

The plasma levels of 5-HT, 5-HIAA, HVA, and MHPG were not associated with age and there was no significant difference in those plasma levels between male and female.

\section{Correlation between plasma 5-HIAA level and total CES-D scales in patients with COPD}

Plasma level of 5-HIAA $(r=0.24, p=0.049)$, but not that of 5 -HT $(r=-0.06, p>0.05)$, HVA $(r=0.19, p>0.05)$, or MHPG $(\mathrm{r}=0.14, \mathrm{p}>0.05)$, was significantly associated with total CES-D scales in patients with COPD (Figure 2).

\section{Correlation between plasma 5-HIAA level and BMI, lung} function, arterial blood gas parameters, and total SGRQ score in patients with COPD

There was a significant correlation between BMI and plasma MHPG $(\mathrm{r}=-0.24, \mathrm{p}=0.041)$, but not 5 -HIAA $(\mathrm{r}=-$ $0.21, \mathrm{p}>0.05), 5-\mathrm{HT}(\mathrm{r}=0.07, \mathrm{p}>0.05)$, HVA $(\mathrm{r}=-0.09$, $\mathrm{p}>0.05)$, and MHPG $(\mathrm{r}=-0.24, \mathrm{p}=0.041)$ level in COPD patients.

Plasma 5-HIAA and MHPG level showed significant negative associations with $\% \mathrm{FVC}, \% \mathrm{FEV}_{1}$, and partial pressure of arterial oxygen $\left(\mathrm{PaO}_{2}\right)$, and positively associations with partial pressure of arterial carbon dioxide $\left(\mathrm{PaCO}_{2}\right)$ and total SGRQ scores. There was no significant correlation between the plasma 5-HT level and lung function, arterial blood gas parameters, or total SGRQ scores. Plasma HVA level showed a significant negative association with $\% \mathrm{FEV}_{1}$ and $\mathrm{PaO}_{2}$, and a positive association with $\mathrm{PaCO}_{2}$ and total SGRQ scores (Table 2).

\section{Correlation between lung function, total SGRQ scores and total CES-D scales in patients with COPD}

The $\% \mathrm{FEV}_{1}$ showed a significant negative association with total SGRQ scores $(\mathrm{r}=-0.69, \mathrm{p}<0.0001)$ and total CES-D scales $(\mathrm{r}=-0.27, \mathrm{p}=0.025)$ in patients with COPD. The $\mathrm{PaO}_{2}$ $(\mathrm{r}=-0.53, \mathrm{p}<0.0001)$ and $\mathrm{PaCO}_{2}(\mathrm{r}=0.44, \mathrm{p}=0.0002)$ showed a significant association with total SGRQ scores. Interestingly, there was no correlation between $\mathrm{PaO}_{2}(\mathrm{r}=-0.21$, $\mathrm{p}>0.05)$ and $\mathrm{PaCO}_{2}(\mathrm{r}=0.22, \mathrm{p}>0.05)$ and total CES-D scales in COPD patients. 


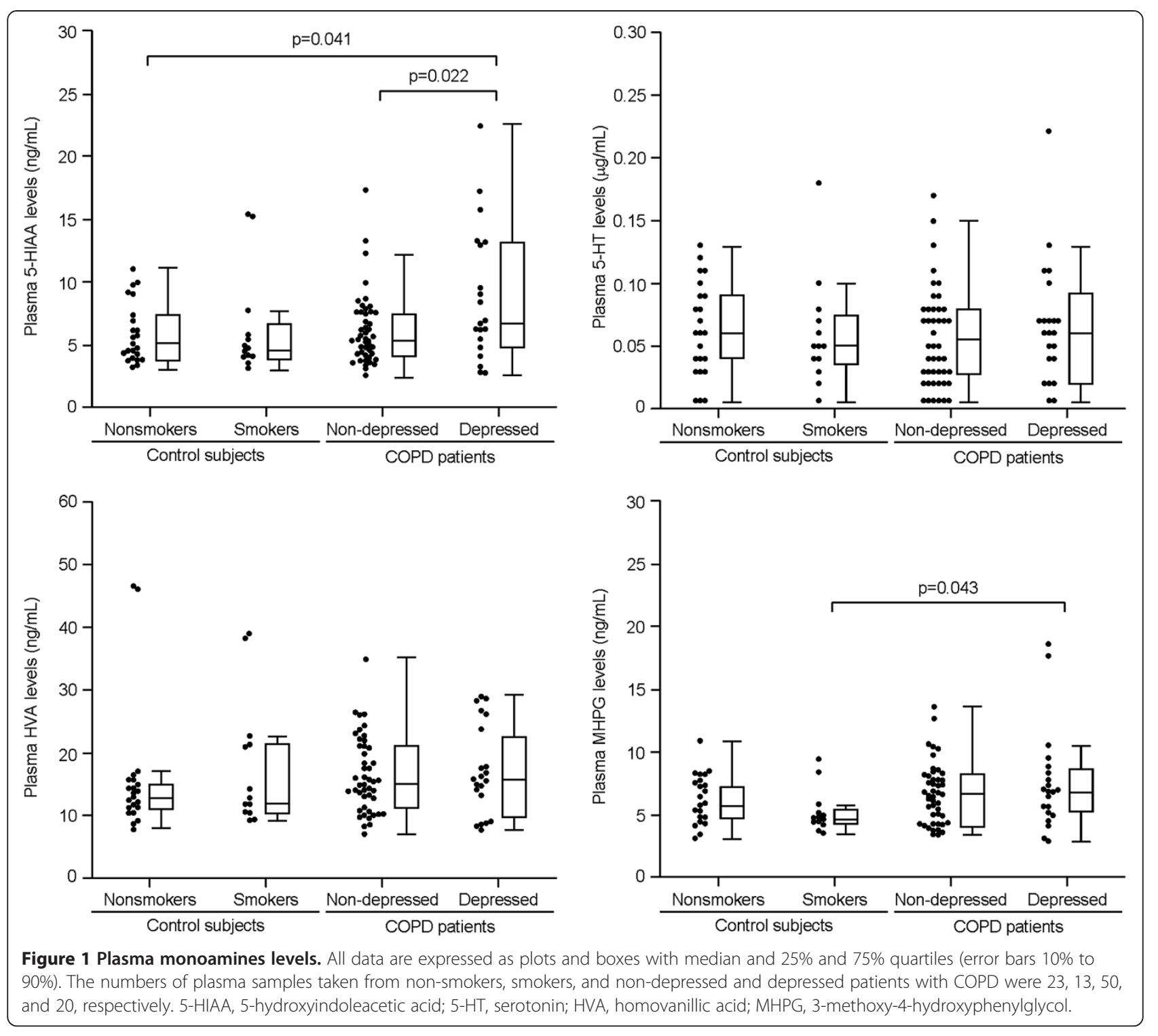

\section{Discussion}

To our knowledge, this is the first study to have measured the plasma levels of monoamines and their metabolites in depressed patients with COPD. Our present results demonstrated that depressed patients with COPD had significantly higher plasma 5-HIAA levels than nondepressed COPD patients and non-smokers. The plasma 5-HIAA levels also showed a significant positive correlation with the severity of depression in the patients with COPD. Our present results support those of a previous study demonstrating that the plasma 5-HIAA levels were significantly increased in patients with depression relative to control subjects, and that the plasma 5-HIAA levels were positively related to the severity of depression [14]. Previous studies have shown that the level of 5-HIAA in cerebrospinal fluid (CSF) was positively associated with the severity of depression in abstinent alcoholics, and that treatment with the antidepressant fluoxetine decreased both the CSF 5-HIAA levels and the mean Hamilton depression rating scale score $[27,28]$. Other studies have demonstrated that decreased CSF 5HIAA levels were associated with attempted suicide in patients with depression, and that non-impulsive suicide attempters had higher plasma 5-HIAA levels than impulsive suicide attempters $[15,29,30]$. In the COPD patients we analyzed, increased plasma 5-HIAA levels were also associated with poor lung function, hypoxia and hypercapnia. Poor lung function is closely correlated with a poor HRQOL, and may result in depression. In COPD patients it has been shown that hypoxia and/or hypercapnia induces oxidative stress and results in an increase of reactive oxygen species (ROS) throughout the whole body including the lungs, brain, and muscles [1-5]. Therefore, it is possible that ROS may have a direct or 

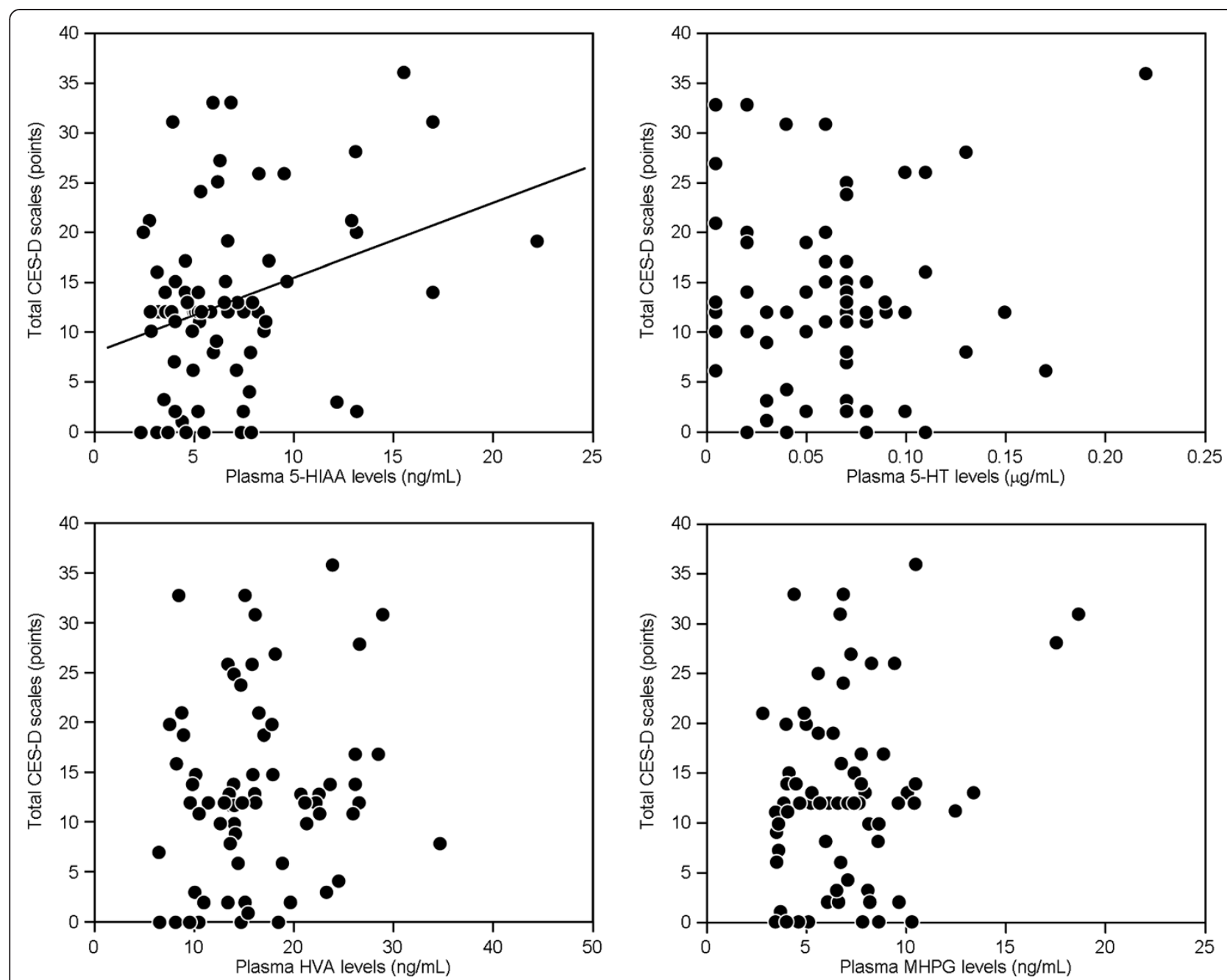

Figure 2 Correlations between total CES-D scales and plasma monoamines levels in patients with COPD. Correlations were analyzed using non-parametric Spearman's test ( $n=70$ ). CES-D, Centre for Epidemiologic Studies Depression scale; 5-HIAA, 5-hydroxyindoleacetic acid; 5-HT, serotonin; HVA, homovanillic acid; MHPG, 3-methoxy-4-hydroxyphenylglycol.

indirect effect on serotonergic innervation of the lungs, and also affect brain serotonin turnover in the respiratory centres/autonomic centres of the brain. Further analysis will be needed to clarify this issue. None of the participants included in our study had previously attempted suicide. It has been reported that the severity of depression in patients with COPD is closely associated with suicidal ideation $[8,9]$. Therefore, it would be worthwhile to rank COPD patients in terms of current suicide risk. Taken together, the data suggest that a depressive status and the severity of COPD may be related to increased plasma 5HIAA level in depressed COPD patients.

Table 2 Correlations between plasma monoamines levels and lung function, arterial blood gas parameters, and total SGRQ scores in the patients with COPD

\begin{tabular}{cccccc}
\hline & \%FVC, \% & \%FEV $_{\mathbf{1}}, \%$ & PaO $_{\mathbf{2}}$, Torr & PaCO $_{2}$, Torr & Total SGRQ, units \\
\hline 5-HIAA, $\mathbf{n g} / \mathbf{m L}$ & $-0.36(0.002)$ & $-0.40(<0.001)$ & $-0.38(<0.001)$ & $0.26(0.031)$ & $0.33(0.006)$ \\
5-HT, $\boldsymbol{\mu g} / \mathbf{m L}$ & $-0.09(\mathrm{NS})$ & $-0.08(\mathrm{NS})$ & $-0.17(\mathrm{NS})$ & $0.06(\mathrm{NS})$ & $0.10(\mathrm{NS})$ \\
$\mathbf{H V A}, \mathbf{n g} / \mathbf{m L}$ & $-0.36(0.002)$ & $-0.49(<0.001)$ & $-0.39(<0.001)$ & $0.09(\mathrm{NS})$ & $0.26(0.029)$ \\
MHPG, $\mathbf{n g} / \mathbf{m L}$ & $-0.21(\mathrm{NS})$ & $-0.46(<0.001)$ & $-0.41(<0.001)$ & $0.25(0.036)$ & $0.40(<0.001)$ \\
\hline
\end{tabular}

All correlation coefficients were expressed as $r$ ( $p$ value).

Post-bronchodilator data for \%FVC and \%FEV 1 were used.

5-HIAA, 5-hydroxyindoleacetic acid; 5-HT, 5-hydroxytryptamine; HVA, homovanillic acid; MHPG, 3-methoxy-4-hydroxyphenylglycol; FVC, forced expiratory capacity; FEV 1 , forced expiratory volume in 1 second; $\mathrm{PaO}_{2}$, partial pressure of oxygen; $\mathrm{PaCO}_{2}$, partial pressure of carbon dioxide; SGRQ, St. George's Respiratory Questionnaire. 
The metabolism of 5-HT is controlled exclusively via the action of monoamine oxidase and aldehyde dehydrogenase, resulting in the formation of 5-HIAA. In this study, we did not find any differences in the plasma 5-HT levels among the four groups (non-smokers, smokers, non-depressed COPD and depressed COPD). However, a previous study demonstrated that the plasma 5-HT levels were increased in patients with COPD and was also associated with aging [31]. Further analysis should be needed to verify this issue.

HVA is a dopamine metabolite. Previous studies of patients with depression have demonstrated that the levels of HVA in plasma and CSF decreased along with the levels of 3 , 4-dihydroxyphenylacetic acid [14,32,33]. In the present study, plasma HVA levels showed no differences among the four groups we examined. However, the plasma level of HVA showed significant negative associations with \%FVC, $\% \mathrm{FEV}_{1}$, and $\mathrm{PaO}_{2}$ levels, and a positive association with the total SGRQ scores in patients with COPD. In COPD patients, most of the HVA in plasma may be derived from precursor dopamine in sympathetic nerves rather than brain dopamine. Our results suggest that poor lung function induced ROS, and perhaps resulted in an increase of HVA derived from precursor dopamine in sympathetic nerves in the COPD patients we studied. Further analysis will be needed to verify this hypothesis.

MHPG is a metabolite of both epinephrine and norepinephrine. Depressed COPD patients had significantly higher plasma MHPG levels ( $\mathrm{p}=0.043)$ than smokers. Placidi and coworkers [29] suggested that the level of MHPG in CSF might have a positive correlation with aggressive and impulsive suicide, and that selective norepinephrine reuptake inhibitors might increase the risk of suicidal acts. In the present study, however, the plasma level of MHPG was not associated with the severity of depression, but showed significant negative associations with \% $\mathrm{FEV}_{1}$ and $\mathrm{PaO}_{2}$, and positive associations with $\mathrm{PaCO}_{2}$ and the total SGRQ score in patients with COPD. Thus, the plasma levels of both HVA and MHPG may be related to the severity of COPD rather than to depression in COPD.

There were some limitations to the present study. First, we measured the levels of monoamines in plasma but not in CSF. Fluctuations in the levels of monoamines and their metabolites can differ between peripheral blood (plasma) and the brain (CSF). Second, the depressed and non-depressed COPD patients were not matched for the severity of COPD, and this parameter may be correlated with plasma monoamine levels. Third, we did not take into account the effects of treatments with antidepressants on the plasma levels of monoamines in depressed COPD patients, although previous studies have reported that antidepressants are of little benefit to patients with COPD $[9,34,35]$. Further analysis of these issues will be necessary.

\section{Conclusion}

In summary, the present study has shown that plasma 5HIAA levels are significantly increased in COPD patients with depression, and also associated with the severity of depression in such patients. We also found that the plasma 5-HIAA, MHPG, and HVA levels were negatively associated with lung function, HRQOL and arterial blood gas abnormalities in patients with COPD. Plasma monoamine levels may be applicable as biomarkers for detection of depression in patients with COPD.

\section{Abbreviations}

ANOVA: Analysis of variance; ATS: American thoracic society; BMI: Body mass index; CES-D: Centre for epidemiologic studies depression; COPD: Chronic obstructive pulmonary disease; CSF: Cerebrospinal fluid; CT: Computed tomography; $\mathrm{FEV}_{1}$ : Forced expiratory volume in 1 second; FVC: Forced vital capacity; GOLD: Global strategy for diagnosis, management, and prevention of COPD; HRCT: High resolution computed tomography; HRQOL: Healthrelated quality of life; HVA: Homovanillic acid; ICS: Inhaled corticosteroid; LABA: Long-acting $\beta_{2}$ agonist; LAMA: Long-acting muscarinic receptor antagonist; MHPG: 3-methoxy-4-hydroxyphenylglycol; MRI: Magnetic resonance imaging; $\mathrm{PaCO}_{2}$ : Partial pressure of arterial carbon dioxide; $\mathrm{PaO}_{2}$ : Partial pressure of arterial oxygen; SD: Standard deviation; SGRQ: St. George's respiratory questionnaire; SRT: Slow-release theophylline; mMRC: Modified medical research council; \%FEV 1 : Percent of predicted forced expiratory volume in 1 second; \%FVC: Percent of predicted forced vital capacity; 5-HIAA: 5-hydroxyindoleacetic acid; 5-HT: Serotonin (5-hydroxytryptamine).

\section{Competing interests}

This work has no financial competing interests. This work was funded by a grant from the Ministry of Health, Labor and Welfare of Japan (KT), and by a Grant-in-Aid for Scientific Research (C) (no. 21590977: T.H.) from the Ministry of Education, Science, Sports, and Culture of Japan.

\section{Authors' contributions}

TS-K contributed to protocol design, data collection, analysis, and writing of the manuscript. TK contributed to protocol design and editing of the manuscript. Kl contributed to data collection. YS supervised the protocol design. KM contributed to data collection. MO contributed to data collection. $\mathrm{NE}$ contributed to data collection. HI contributed to data collection. NU supervised the protocol design and edited the manuscript. TH supervised the protocol design and edited the manuscript. All authors read and approved the final manuscript.

\section{Acknowledgement}

The authors extend special thanks to Professor Hisamichi Aizawa, MD, PhD, Kurume University School of Medicine, for contributing to the protocol design, Tatsuyuki Kakuma, PhD, Professor of Biostatistics, , Biostatistics center, Kurume University, for analysis of statistics, Kyoko Yamagichi, BSc, and Emiko Kuma, BSc, Kurume University School of Medicine, for technical support, and Koichi Nishimura, MD, PhD, Nagoya Kyouritsu Hospital, for technical support with the Japanese validated SGRQ. The authors are also grateful to Masaharu Kinoshita, MD, PhD, and Reiko Toda, MD, PhD, Yanagawa Nagata Hospital, and Tatsuya Mukaino, MD, and Keita Matsumoto, MD, Social Insurance Tagawa Hospital, for collection of data on the study subjects.

\section{Author details}

${ }^{1}$ Division of Respirology, Neurology, and Rheumatology, Department of Medicine 1, Kurume University School of Medicine, 67 Asahi-machi, Kurume 830-0011, Japan. ${ }^{2}$ Department of Neuropsychiatry, Kurume University School of Medicine, 67 Asahi-machi, Kurume 830-0011, Japan.

Received: 10 October 2012 Accepted: 28 May 2013

Published: 31 May 2013

\section{References}

1. Mannino DM: COPD: epidemiology, prevalence, morbidity and mortality, and disease heterogeneity. Chest 2002, 121(5 Suppl):121S-126S. 
2. Global Initiative for Chronic Obstructive Lung Disease (GOLD). Global Strategy for the Diagnosis, Management, and Prevention of Chronic Obstructive Pulmonary Disease NHLBI/WHO Workshop report. Rev. ed: ; 2011. http://www.goldcopd.com.

3. Ito K, Kawayama T, Shoji Y, Fukushima N, Matsunaga K, Edakuni N, Uchimura N, Hoshino T: Depression, but not sleep disorder, is an independent factor affecting exacerbations and hospitalization in patients with chronic obstructive pulmonary disease. Respirology 2012, 17(6):940-949.

4. Schneider $C$, Jick SS, Bothner U, Meier CR: COPD and the risk of depression. Chest 2010, 137(2):341-347.

5. Xu W, Collet JP, Shapiro S, Lin Y, Yang T, Platt RW, Wang C, Bourbeau J: Independent effect of depression and anxiety on chronic obstructive pulmonary disease exacerbations and hospitalizations. Am J Respir Crit Care Med 2008, 178(9):913-920.

6. Fan VS, Ramsey SD, Giardino ND, Make BJ, Emery CF, Diaz PT, Benditt JO, Mosenifar Z, McKenna R Jr, Curtis JL, et al: Sex, depression, and risk of hospitalization and mortality in chronic obstructive pulmonary disease. Arch Intern Med 2007, 167(21):2345-2353.

7. Lewis KE, Annandale JA, Sykes RN, Hurlin C, Owen C, Harrison NK: Prevalence of anxiety and depression in patients with severe COPD: similar high levels with and without LTOT. COPD 2007, 4(4):305-312.

8. Goodwin RD: Is COPD associated with suicide behavior? J Psychiatr Res 2011, 45(9):1269-1271.

9. Lacasse $Y$, Beaudoin $L$, Rousseau L, Maltais F: Randomized trial of paroxetine in end-stage COPD. Monaldi Arch Chest Dis 2004, 61(3):140-147.

10. Hindmarch I: Expanding the horizons of depression: beyond the monoamine hypothesis. Hum Psychopharmacol 2001, 16(3):203-218.

11. Anderson GM: Peripheral and central neurochemical effects of the selective serotonin reuptake inhibitors (SSRIs) in humans and nonhuman primates: assessing bioeffect and mechanisms of action. Int J Dev Neurosci 2004, 22(5-6):397-404.

12. Lewis A, Miller JH, Lea RA: Monoamine oxidase and tobacco dependence. Neurotoxicology 2007, 28(1):182-195

13. Logan J, Fowler JS: Evidence for reduced arterial plasma input, prolonged lung retention and reduced lung monoamine oxidase in smokers. Nucl Med Biol 2005, 32(5):521-529.

14. Mitani H, Shirayama Y, Yamada T, Kawahara R: Plasma levels of homovanillic acid, 5-hydroxyindoleacetic acid and cortisol, and serotonin turnover in depressed patients. Prog Neuropsychopharmacol Biol Psychiatry 2006, 30(3):531-534.

15. Spreux-Varoquaux O, Alvarez JC, Berlin I, Batista G, Despierre PG, Gilton A, Cremniter D: Differential abnormalities in plasma 5-HIAA and platelet serotonin concentrations in violent suicide attempters: relationships with impulsivity and depression. Life Sci 2001, 69(6):647-657.

16. American Thoracic Society: Standardization of Spirometry, 1994 Update. Am J Respir Crit Care Med 1995, 152(3):1107-1136.

17. Toda R, Hoshino T, Kawayama T, Imaoka H, Sakazaki Y, Tsuda T, Takada S, Kinoshita M, Iwanaga T, Aizawa H: Validation of "lung age" measured by spirometry and handy electronic FEV1/FEV6 meter in pulmonary diseases. Intern Med 2009, 48(7):513-521.

18. Hajiro T, Nishimura K, Tsukino M, Ikeda A, Koyama H, Izumi T: Comparison of discriminative properties among disease-specific questionnaires for measuring health-related quality of life in patients with chronic obstructive pulmonary disease. Am J Respir Crit Care Med 1998, 157(3 Pt 1):785-790.

19. Jones PW, Quirk FH, Baveystock CM, Littlejohns P: A self-complete measure of health status for chronic airflow limitation. The St. George's Respiratory Questionnaire. Am Rev Respir Dis 1992, 145(6):1321-1327.

20. Bestall JC, Paul EA, Garrod R, Garnham R, Jones PW, Wedzicha JA: Usefulness of the Medical Research Council (MRC) dyspnoea scale as a measure of disability in patients with chronic obstructive pulmonary disease. Thorax 1999, 54(7):581-586

21. Goddard PR, Nicholson EM, Laszlo G, Watt I: Computed tomography in pulmonary emphysema. Clin Radiol 1982, 33(4):379-387.

22. Iwata N, Saito K, Roberts RE: Responses to a self-administered depression scale among younger adolescents in Japan. Psychiatry Res 1994, 53(3):275-287.

23. Zigmond AS, Snaith RP: The hospital anxiety and depression scale. Acta Psychiatr Scand 1983, 67(6):361-370.

24. Nebinger $P$, Koel $M$ : Automatic determination of serotonin in biological fluids by liquid chromatography. J Chromatogr 1988, 427(2):326-330.
25. Nagaoka S, Iwamoto N, Arai H: First-episode neuroleptic-free schizophrenics: concentrations of monoamines and their metabolites in plasma and their correlations with clinical responses to haloperidol treatment. Biol Psychiatry 1997, 41(8):857-864.

26. Hanai J, Kawai T, Sato Y, Takasugi N, Nishi M, Takeda T: Simple liquidchromatographic measurement of vanillylmandelic acid and homovanillic acid in urine on filter paper for mass screening of neuroblastoma in infants. Clin Chem 1987, 33(11):2043-2046.

27. Heinz A, Weingartner $H$, George D, Hommer D, Wolkowitz OM, Linnoila M: Severity of depression in abstinent alcoholics is associated with monoamine metabolites and dehydroepiandrosterone-sulfate concentrations. Psychiatry Res 1999, 89(2):97-106.

28. De Bellis MD, Geracioti TD Jr, Altemus M, Kling MA: Cerebrospinal fluid monoamine metabolites in fluoxetine-treated patients with major depression and in healthy volunteers. Biol Psychiatry 1993, 33(8-9):636-641.

29. Placidi GP, Oquendo MA, Malone KM, Huang YY, Ellis SP, Mann JJ: Aggressivity, suicide attempts, and depression: relationship to cerebrospinal fluid monoamine metabolite levels. Biol Psychiatry 2001, 50(10):783-791.

30. Mann JJ, Malone KM, Sweeney JA, Brown RP, Linnoila M, Stanley B, Stanley M: Attempted suicide characteristics and cerebrospinal fluid amine metabolites in depressed inpatients. Neuropsychopharmacol 1996, 15(6):576-586

31. Lau WK, Chan-Yeung MM, Yip BH, Cheung AH, Ip MS, Mak JC: The role of circulating serotonin in the development of chronic obstructive pulmonary disease. PLoS One 2012, 7(2):e31617.

32. Engstrom G, Alling C, Blennow K, Regnell G, Traskman-Bendz L: Reduced cerebrospinal HVA concentrations and HVA/5-HIAA ratios in suicide attempters. Monoamine metabolites in 120 suicide attempters and 47 controls. Eur Neuropsychopharmacol 1999, 9(5):399-405.

33. Kapur S, Mann JJ: Role of the dopaminergic system in depression. Biol Psychiatry 1992, 32(1):1-17.

34. Yohannes AM, Connolly MJ: Do antidepressants work in patients with chronic obstructive pulmonary disease with comorbid depression? Expert Rev Respir Med 2011, 5(6):727-729.

35. Yohannes AM, Connolly MJ, Baldwin RC: A feasibility study of antidepressant drug therapy in depressed elderly patients with chronic obstructive pulmonary disease. Int J Geriatr Psychiatry 2001, 16(5):451-454

\section{doi:10.1186/1471-244X-13-159}

Cite this article as: Sekiduka-Kumano et al:: Positive association between the plasma levels of 5-hydroxyindoleacetic acid and the severity of depression in patients with chronic obstructive pulmonary disease. BMC Psychiatry 2013 13:159.

\section{Submit your next manuscript to BioMed Central and take full advantage of:}

- Convenient online submission

- Thorough peer review

- No space constraints or color figure charges

- Immediate publication on acceptance

- Inclusion in PubMed, CAS, Scopus and Google Scholar

- Research which is freely available for redistribution 